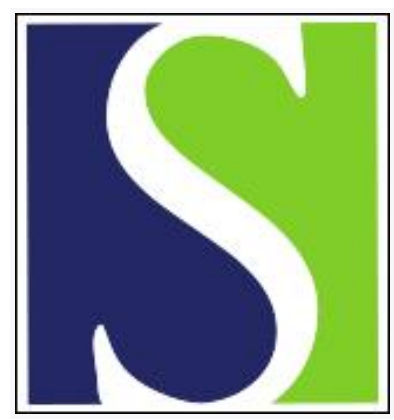

Scand J Work Environ Health 2005;31(2):138-151

https://doi.org/10.5271/sjweh.861

Issue date: Apr 2005

Task-based estimation of mechanical job exposure in occupational groups

by Mathiassen SE, Nordander C, Svendsen SW, Wellman HM, Dempsey PG

Affiliation: Centre for Musculoskeletal Research, University of Gävle, Box 7629, SE-90712 Umeå, Sweden. svenderik.mathiassen@hig.se

The following articles refer to this text: $2009 ; 35(2): 96-112$;

2015;41(1):65-74

Key terms: estimation bias; exposure variability; job-exposure matrix; mechanical job exposure; muscle activity; occupational epidemiology; occupational group; occupational group mean; task-based estimation; task-based model

This article in PubMed: www.ncbi.nlm.nih.gov/pubmed/15864908

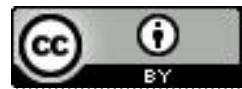




\title{
Task-based estimation of mechanical job exposure in occupational groups
}

\author{
by Svend Erik Mathiassen, PhD, ${ }^{1,2}$ Catarina Nordander, MD, ${ }^{3}$ Susanne W Svendsen, PhD, ${ }^{4}$ Helen M \\ Wellman, MS, ${ }^{2}$ Patrick G Dempsey, $P h D^{2}$
}

\begin{abstract}
Mathiassen SE, Nordander C, Svendsen SW, Wellman HM, Dempsey PG. Task-based estimation of mechanical job exposure in occupational groups. Scand J Work Environ Health 2004;31(2):138-151.
\end{abstract}

\begin{abstract}
Objectives This study examined the validity of a common belief in epidemiology with respect to work-related musculoskeletal disorders, that individual mechanical job exposure is better estimated from tasks performed in the job than from the mean exposure of the occupational group.

Methods Whole-day recordings of upper trapezius electromyography were obtained from 24 cleaners and 23 office workers. Trapezius activity was analyzed in the level (gap time) and frequency (jerk time) dimensions. On the same day, the job of each person was divided into periods of active work and breaks by means of continuous observations. The bootstrap re-sampling technique was used with this database to compare task-based jobexposure estimates with estimates based on the occupational mean. For a particular person, the task-based estimate was obtained by combining the average work and break exposures in the occupation with the personal time proportions of the two tasks in the job.

Results The task-based estimates were, in general, equivalent to, or less correct than, occupation-based estimates for both exposure parameters in both occupations and for individual exposures, as well as for group means. This was the result in spite of significant and consistent exposure differences between work and breaks, in particular among the cleaners.

Conclusions Even if task exposure contrasts are large, task-based estimates of job exposures can be less correct than estimates based on the occupational mean. Since collecting and processing task information is costly, it is recommended that task-based modeling of mechanical exposure be implemented in studies only after careful examination of its possible benefits.
\end{abstract}

Key terms estimation bias; exposure variability; job-exposure matrix; muscle activity; occupational epidemiology; occupational group mean; task-based model.

Correct exposure data are vital to studies that compare the exposure of different groups or conditions and that investigate relationships between exposure and outcome. Systematic errors of the mean (ie, bias) can lead to spurious results irrespective of the type of study. Errors that cause increased dispersion of data around the mean decrease statistical power in intervention studies $(1,2)$ and usually attenuate exposure-outcome regressions in epidemiologic studies (3-5).

Several reviews addressing work-related musculoskeletal disorders have emphasized that strategies for assessing physical workload (mechanical exposure) are often insufficient (6-10). One important point of criticism concerns the common approach of estimating the job exposure of a worker by the mean exposure of his or her occupational group, even though mechanical job exposures are known to differ largely between persons working in the same occupation.

Some researchers have proposed that mechanical job-exposure estimates would be more correct if based on tasks occurring in jobs rather than on merely occupation $(6,10,11)$. For instance, the job exposure of a particular person could be estimated by combining information on the proportions of all different tasks in the job of that person with expected task exposures according to a database derived with other persons (6). Thus the job-exposure estimate would be based on a taskexposure matrix (12) rather than on a job-exposure

1 Centre for Musculoskeletal Research, University of Gävle, Umeå, Sweden.

2 Liberty Mutual Research Institute for Safety, Hopkinton, Massachusetts, United States.

3 Department of Occupational and Environmental Medicine, Lund University Hospital, Lund, Sweden.

4 Department of Occupational Medicine, University Hospital of Aarhus, Aarhus, Denmark.

Reprint requests to: Dr Svend Erik Mathiassen, Centre for Musculoskeletal Research, University of Gävle, Box 7629, SE90712 Umeå, Sweden. [E-mail: svenderik.mathiassen@hig.se] 
matrix $(13,14)$. A task-based modeling approach has been suggested for several occupational and environmental exposures (15-19). As concerns mechanical exposures, task-based modeling has been used to assess energy expenditure at work $(20,21)$, vibration (22), work postures (23-25), and low-back load in existing (26) and hypothetical jobs (27). These studies represent several different purposes, for instance, the determination of exposure-outcome relationships and the evaluation of ergonomic control measures.

However, the correctness of task-based estimates of mechanical job exposures has rarely been investigated. Since task-based exposure assessment usually requires substantially more resources for data collection and analysis than an assessment based only on occupation, it is important to know whether it does, indeed, result in better estimates. To our knowledge, only one study has pursued that issue, and it indicated a limited usefulness of a task-exposure matrix for estimating upper-arm postures in three different occupations (28). Furthermore, Mathiassen et al (29) recently reported very small gains in efficiency from using task-based procedures for sampling mechanical exposures during one measurement day, as compared with random sampling without consideration to tasks. A study by Pernold et al (21) suggested that job energy expenditure could be well estimated by a model based on task exposures. However, the persons represented a wide range of occupations, and the study did not investigate whether a simple job-exposure matrix would have been equally successful. Thus the available evidence, while limited, does not suggest any clear advantage of task-based assessment of mechanical job exposures over models based only on occupation.

To investigate this issue further, we have assessed the correctness of job-exposure estimates based on a task-exposure matrix combined with personal task proportions and compared it to estimates based on a jobexposure matrix. The study used data on upper trapezius electromyography from a larger study of mechanical exposure and musculoskeletal disorders among office workers and cleaners (30-32).

\section{Study population and methods}

\section{Parent data set}

Thirty-two office workers and twenty-five hospital cleaners participated in the original study. The two occupations were intended to represent jobs with long periods at low loads (office workers), and less uniform, yet larger load levels [cleaners $(29,30)]$. Electromyography (EMG) was recorded from the right upper trapezius muscle of each participant for one complete workday, excluding the lunch break, by means of portable data loggers (33).
The recording preparations, signal acquisition, and basic data processing have been described elsewhere (30, $31,34)$. Only workers with acceptable EMG recordings for more than $90 \%$ of the day were included in the final study population, which was comprised of 24 cleaners and 23 office workers (29). For each of these persons, the data file consisted of root-mean-square-converted EMG amplitude values at $8 \mathrm{~Hz}$, normalized to the EMG amplitude obtained during a maximal voluntary exertion (\%MVE).

During the entire measurement day, excluding the lunch break, the worker was followed by a researcher, who assigned activities to one of the following eight exhaustive task categories (35): cleaning small areas, cleaning large areas, maintenance, transportation, computer work, other office tasks, meetings, and breaks (ie, any periods of activity or rest that could be discriminated from work). Since a previous study had shown marginal contrasts in trapezius EMG between tasks performed during actual work in both occupations (29), further analysis was restricted to the two major task categories of breaks (as above) and work (all other tasks in the job).

Each person's EMG file was split into periods of work and breaks according to the observational records, and the overall proportion of time in either category was determined. Separate exposure variation analyses (EVA) (36) were performed on the data within each of the two task categories and for the entire job EMG recording, without regard to task. The following two parameters were extracted from the analyses, as described earlier (29): the proportion of time spent at an amplitude level below 1\% MVE ("gap time") and the proportion of time when the EMG amplitude remained within the same amplitude category in periods shorter than 1 second ("jerk time"). Gap time expresses the occurrence of loads at low levels, large values indicating more "muscular rest" (30, 31, 37-39). Jerk time measures the frequency dimension of exposure, large values indicating frequent shifts in EMG amplitude (29). Thus, for a particular person $i$, data were available on mean gap time and jerk time at an overall job level, $m_{j i}$, and within the task categories work and breaks, $m_{t i}$; as well as on the time proportions, $W_{t i}$, of the two task categories in the job. Obviously, for both exposure parameters: $m_{j i}=$ $\Sigma_{t i}\left[W_{t i} \times m_{t i}\right]$, where $\Sigma_{t i}$ indicates summation across tasks in the job of person $i$.

Within each occupational group and for both task categories, Pearson correlation coefficients were determined between the task proportion and either one of the parameters gap time and jerk time. For both exposure parameters, the contrast between work and breaks was assessed using an omega-square coefficient, $C_{T}$, which can be regarded as the fixed-effect analogy of an intraclass correlation (40):

$C_{T}=M S E_{T} /\left(M S E_{T}+S_{S t}^{2}\right)$, equation 1 
where $M S E_{T}=$ mean squared error between the two task categories, $s_{S t}^{2}=$ overall exposure variance between subjects within task, including within-subject components.

$C_{T}$ can assume values between 0 and 1 , the former reflecting no exposure contrast between tasks and the latter showing that any possible difference in job exposure between persons can be completely explained by different task proportions. Input data for equation 1 were obtained using standard analysis of variance (ANOVA) algorithms for fixed-effect one-way designs (40). Finally, true individual job exposures were linear least-square regressed on their task-based estimates, as obtained by multiplying personal proportions of time in work and breaks by the mean occupational group exposure in either task category and adding the two products.

\section{Job-exposure estimation}

According to the task-exposure-matrix (TEM) model, the mean job exposure of person $i$ was estimated by combining his or her personal task proportions with task exposures obtained from a task-exposure matrix based on other persons within the same occupation:

$$
m_{j i t}=\Sigma_{t i}\left[W_{t i} \times m_{t}\right],
$$

equation 2

where $m_{j i l t}=$ the job-exposure estimate according to the TEM model, $W_{t i}=$ the proportion of task $t$ in the job of person $i, m_{t}=$ exposure of task $t$ in the task-exposure matrix, $\Sigma_{t i}=$ summation across tasks in the job of person $i$.

For comparison, job exposure was also estimated using a job-exposure-matrix (JEM) model. In that model, the estimated job exposure $m_{j i l j}$ of person $i$ equaled the mean job exposure in a database of persons with the same occupation as $i$ :

$$
m_{j i j}=m_{j},
$$

equation 3

where $m_{j}=$ occupational mean job exposure in the database.

\section{Performance of the estimation models}

In order to simulate the situation of job exposures in a "target" sample being estimated on the basis of an exposure database derived with other persons within the same occupation, a bootstrap technique was applied (1, $41,42)$. Bootstrapping generates an empirical distribution of some experimental target parameter through a large number of virtual replications of the original experiment. For this purpose, data are re-sampled with replacement from the parent dataset. Bootstrapping offers an attractive alternative to other simulation techniques, in particular when parent data distributions are difficult to parametrize.

Thus the following procedure was repeated 2000 times for each of 6 combinations of occupation (cleaners, office workers) and number of persons in the exposure database $\left(n_{b}=4,8,12\right)$. These sizes of $n_{b}$ rep- resent a typical order of magnitude for occupational studies of trapezius EMG $(1,43)$.

Construction of the job-exposure and task-exposure matrices. From the parent dataset, $n_{b}$ persons were randomly selected with replacement. In this database sample, the mean gap time and jerk time were determined for the entire job, as well as for each of the task categories work and breaks, by averaging individual means (ie, according to a "mean-of-means" approach) (44).

Target sample and exposure estimation for the target sample. Independent of the database sample, another sample of 10 persons was selected with replacement from the parent dataset. Due to the nature of bootstrapping, a particular person could be elected for both this target sample and the database. For each person in the target sample, estimates of the mean gap time and jerk time in the job were obtained according to the JEM and TEM models [ie, $m_{j i l j}$ and $m_{j i l t}$ (see equations 2 and 3)].

Correctness of individual estimates. The correctness of the estimate for person $i$, noted $I_{j i}$ and $I_{t i}$ for the JEM and TEM models, respectively, was measured by the distance between the estimated and true job exposure. For instance, for the JEM model:

$$
I_{j i}=m_{j i l j}-m_{j i},
$$
equation 4

where $m_{\mathrm{ji}}=$ the true job exposure of person $i$.

Correctness of the estimated mean. For both models, the correctness of the estimated group mean, noted $A_{j}$ and $A_{t}$, respectively, was assessed using an approach similar to that used for individual persons. For the JEM model:

$$
\begin{aligned}
A_{j}=\left(1 / n_{e}\right) \times \Sigma_{\mathrm{i}}\left[m_{j i l j}\right]-\left(1 / n_{e}\right) \times \Sigma_{\mathrm{i}}\left[m_{\mathrm{j} j}\right]=\left(1 / n_{e}\right) \times \Sigma_{\mathrm{i}}\left[I_{j i}\right], & \text { equation } 5
\end{aligned}
$$

where $n_{e}=$ the size of the target sample (ie, 10 in this study); $\Sigma_{i}=$ summation across persons in the target sample.

Assessment of gains. For the TEM model, absolute gains in individual correctness, noted $G\left(I_{t i}\right)$, were calculated as:

$$
G\left(I_{t i}\right)=\operatorname{abs}\left(I_{j i}\right)-\operatorname{abs}\left(I_{t i}\right),
$$

equation 6

where abs $=$ the absolute value. Gain in the correctness of the mean, noted $G\left(A_{t}\right)$, was assessed using a similar approach:

$$
G\left(A_{t}\right)=\operatorname{abs}\left(A_{j}\right)-\operatorname{abs}\left(A_{t}\right) .
$$

Thus negative gain values indicate that the TEM model performed worse than the JEM model, while positive values indicate that the TEM model led to more correct estimates. A value of 0 showed that the TEM and JEM models were equally correct. 
For each combination of occupational group $(\mathrm{N}=2)$, the size of the database sample $(\mathrm{N}=3)$, the exposure parameter $(\mathrm{N}=2)$, and the exposure estimation model $(\mathrm{N}=2)$, the bootstrap procedure generated 20000 individual estimates and 2000 group mean estimates for job exposure. Using this dataset, we examined the empirical distributions of estimation correctness in the JEM and TEM models, as well as of the gains associated with the TEM model. In addition, the relationships between TEM and JEM correctness at the individual level were investigated using graphic inspection and linear leastsquare regression.

\section{Results}

\section{Parent dataset}

None of the job-exposure distributions of gap time and jerk time in the two occupational groups differed significantly from normal (Kolmogorov-Smirnoff test, $\mathrm{P}>0.10$ ). However, gap time among the cleaners was positively skewed (skewness 0.86 ), while some negative skewness was found for jerk time among the cleaners $(-0.69)$ and the office workers $(-0.28)$. At the job level, the cleaners and office workers differed significantly in gap time $(\mathrm{P}=0.005$, two-tailed t-test, independent samples) and jerk time $(\mathrm{P}<0.001)$, as discussed in previous publications examining this material $(29,30)$. Both occupations exhibited distinct differences in exposure between work and breaks (table 1), with P-values of 0.02 for jerk time in the office group and $<0.001$ for jerk time in the cleaner group and for gap time in both groups (two-tailed t-test, paired samples). The direction of the difference was, in most cases, consistent at the individual level (figure 1). Thus work was associated

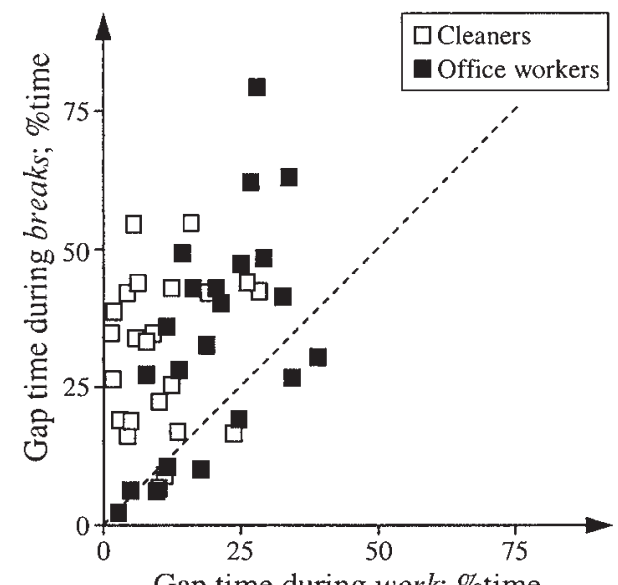

Gap time during work; \%time
Table 1. Size of the parent dataset and exposures to gap time and jerk time among the cleaners and office workers-data for the entire job, as well as for the task categories work and breaks. $\left[n_{s}, N_{q}=\right.$ total number of participants and recorded minutes of exposure, respectively; $w_{t}=$ time proportion of task in the job, mean across all participants in the occupational group; $s_{w t}=$ between-subjects standard deviation of $w_{t} ; m_{t}=$ mean task (job) exposure among the participants performing the task (job), \%time; $s_{S}=$ observed between-subjects standard deviation of exposure, \%time; $C_{T}=$ exposure contrast between tasks (see equation 1); $r\left[m_{w} m_{b}\right]=$ correlation between exposures during work and breaks; $r\left[w_{t} m_{t}\right]=$ correlation between task exposure and time proportion of the task; Slope, Intercept and $r^{2}=$ parameters of the linear least-square regression of true job exposures on the taskbased estimates (see equation 2)]

\begin{tabular}{|c|c|c|c|c|c|c|}
\hline & \multicolumn{3}{|c|}{ Cleaners } & \multicolumn{3}{|c|}{ Office workers } \\
\hline & Job & Work & Breaks & Job & Work & Breaks \\
\hline \multicolumn{7}{|l|}{ Breaks } \\
\hline$n_{s}$ & 24 & 24 & 23 & 23 & 23 & 23 \\
\hline$N_{q}$ & 8861 & 7728 & 1133 & 8612 & 7765 & 847 \\
\hline$w_{t}$ & . & 0.87 & 0.13 & • & 0.90 & 0.10 \\
\hline$s_{w t}$ & . & 0.07 & 0.07 & . & 0.05 & 0.05 \\
\hline \multicolumn{7}{|l|}{ Gap time } \\
\hline$m_{t}$ & 13.8 & 10.7 & 31.0 & 20.7 & 19.6 & 32.4 \\
\hline$s_{S}$ & 7.1 & 7.8 & 13.8 & 10.3 & 10.2 & 20.2 \\
\hline$C_{T}$ & 0.44 & . & $\cdot$ & 0.13 & . & . \\
\hline$r\left[m_{w} m_{b}\right]$ & 0.14 & . & $\cdot$ & 0.58 & . & . \\
\hline$r\left[w_{t} m_{t}\right]$ & $\cdot$ & 0.29 & 0.52 & $\cdot$ & 0.01 & -0.04 \\
\hline Slope & 0.3 & . & . & -0.3 & . & . \\
\hline Intercept & 10 & . & . & 28 & . & . \\
\hline$r^{2}$ & 0.003 & . & . & $<0.001$ & . & . \\
\hline \multicolumn{7}{|l|}{ Jerk time } \\
\hline$m_{t}$ & 66.4 & 70.1 & 41.8 & 41.6 & 42.0 & 37.7 \\
\hline$s_{S}$ & 4.4 & 5.4 & 7.4 & 5.5 & 5.6 & 8.7 \\
\hline$C_{T}$ & 0.83 & . & . & 0.06 & . & . \\
\hline$r\left[m_{w} m_{b}\right]$ & -0.03 & . & . & 0.43 & . & . \\
\hline$r\left[w_{t} m_{t}\right]$ & $\cdot$ & -0.38 & -0.25 & $\cdot$ & 0.11 & -0.24 \\
\hline Slope & 0.1 & . & . & 3.1 & . & . \\
\hline Intercept & 57 & . & . & -89 & . & . \\
\hline$r^{2}$ & 0.005 & . & . & 0.016 & . & . \\
\hline
\end{tabular}

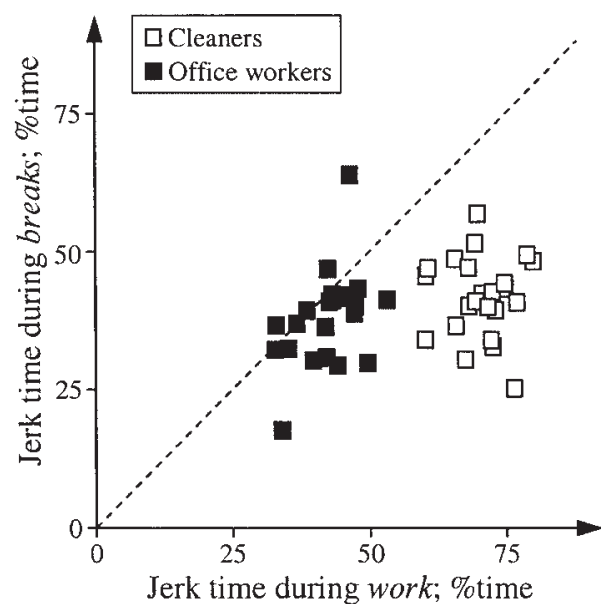

Figure 1. Gap time and jerk time during work and breaks among the cleaners $(N=23)$ and office workers $(N=23)$ in the parent dataset. One cleaner was omitted since her entire workday was classified as work. (broken line = line of identity) 
with less gap time than breaks were for 21 (of 24) cleaners and 16 (of 23) office workers, while all the cleaners and 20 office workers had more jerk time during work than during breaks (figure 1$)$. The task contrasts $\left(C_{T}\right)$ attained substantially lower values for the office workers, however, than for the cleaners due to a smaller exposure difference between work and breaks in the office group and a larger exposure variability within tasks. A tendency for persons being classified as generally low- or high-exposed across tasks was found for the office workers, as indicated by the positive correlation between exposures during work and breaks (table 1). Among the cleaners, clear indications were found of a relationship between task proportion and task exposure for both work and breaks (table 1) (ie, more gap time and less jerk time if the task occurred more frequently).

\section{Job-exposure-matrix model}

Individual exposures. According to the bootstrap simulation, the JEM model led, in median, to a correct estimate of individual gap time among office workers (figure 2).
Gap time among cleaners was overestimated, while jerk time in both groups was, in median, slightly underestimated (figure 2), probably as a result of the skewed distributions of these three job-exposure parameters in the parent dataset. The root-mean-square estimation error (table 2 ) agreed within $0.1 \%$ time with analytical expectations based on the assumption that estimation was unbiased and that $I_{j i}$ was distributed as a difference between two independent variables, in casu the group mean exposure in the database, and the true individual job exposure on one particular day (see equation 4). In that case, the standard deviation of $I_{j i}, s_{I}$, will take the value:

$$
s_{I}=\left(s_{S b}^{2} / n_{b}+s_{S e}^{2}\right)^{1 / 2},
$$

equation 8

where $s_{S b}, s_{S e}=$ the standard deviation for the overall job exposure between subjects in the database and target populations, respectively, and $n_{b}=$ the size of the database sample. In the present case, both $s_{S b}$ and $s_{S e}$ equaled the overall between-subjects dispersion in the parent dataset, that is $s_{S}$ (see table 1), since the database and target samples were both drawn from this source. As $n_{b}$ increased, the distribution of $I_{j i}$ approached a

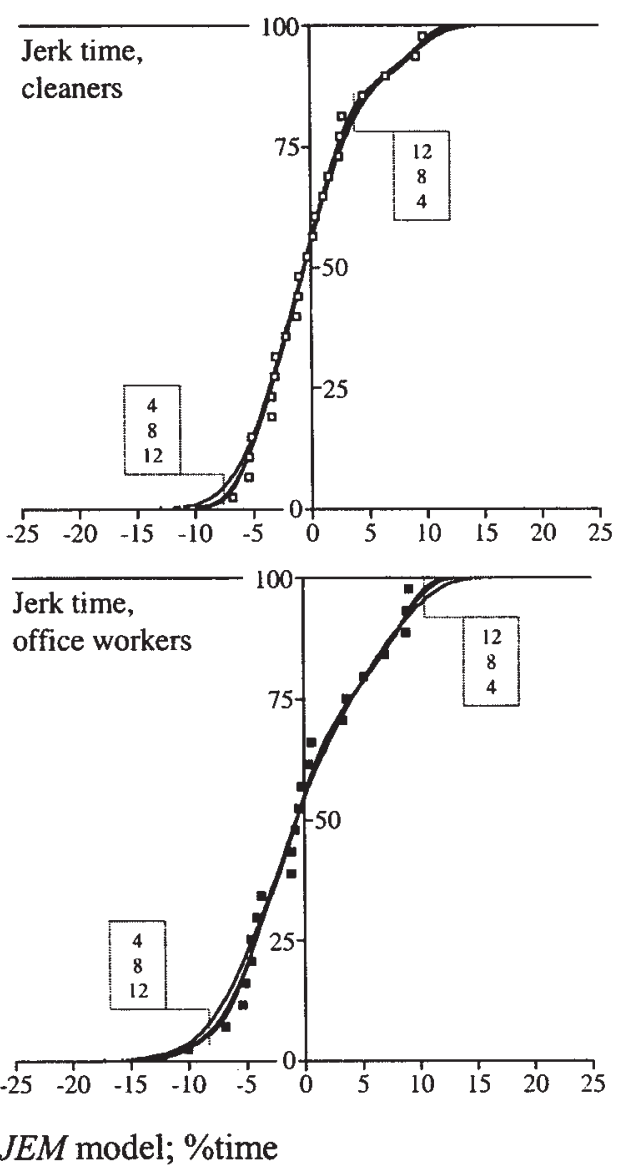

Figure 2. Empirical cumulative bootstrap distributions of the correctness of the job-exposure-matrix (JEM) estimates of individual job exposures (ie, $l_{j i}$; equation 4). (text boxes and connected lines = locations of the distributions from top to bottom; $4,8,12=$ size of the database, $n_{b}$; squares $=$ the cumulative distributions of true individual job exposures in the parent dataset after centering on the mean and change of sign) 
smoothed form of the discrete individual job-exposure distribution in the parent dataset after centering on the group mean and change of sign (figure 2).

Since the distributions of $I_{j i}$ were wide (figure 2), a particular estimate could deviate considerably from the truth, especially in the office group and for gap time. Thus the probability of obtaining an estimate within $\pm 5 \%$ of the time from the truth was about $50 \%$ (gap time) and $73 \%$ (jerk time) for the cleaners, but only $30 \%$ and $58 \%$ for the same parameters, respectively, for the office workers.

Group mean exposures. In contrast to the JEM estimates of individual job exposures, the group means were, in median, estimated very correctly for both occupations and for both parameters (figure 3). However, cases of pronounced incorrectness were again frequent, in particular for gap time, as shown by the root-mean-square (table 2) and empirical distributions (figure 3) of $A_{j}$. In general, the JEM model led to less deviation from true mean exposures among the cleaners than among the office workers, and it performed better for jerk time than for gap time within either group (figure 3). An increase

Table 2. Empirical root-mean-square (rms) errors when the jobexposure-matrix (JEM) and task-exposure-matrix (TEM) models were used for estimating individual job exposures and group means-presented according to occupational group, exposure parameter, and size of the database $\left(n_{b}\right)$.

\begin{tabular}{|c|c|c|c|c|c|c|}
\hline & \multicolumn{3}{|c|}{ Cleaners } & \multicolumn{3}{|c|}{ Office workers } \\
\hline & $n_{b}=4$ & $n_{b}=8$ & $n_{b}=12$ & $n_{b}=4$ & $n_{b}=8$ & $n_{b}=12$ \\
\hline \multicolumn{7}{|l|}{ Gap time } \\
\hline $\operatorname{rms}\left(l_{j i}\right)^{\mathrm{a}}$ & 7.7 & 7.3 & 7.2 & 11.2 & 10.6 & 10.5 \\
\hline $\operatorname{rms}\left(A_{i}\right){ }^{\mathrm{b}}$ & 4.1 & 3.4 & 3.0 & 6.1 & 4.8 & 4.3 \\
\hline $\operatorname{rms}\left(I_{t i}\right)^{c}$ & 7.8 & 7.4 & 7.3 & 11.3 & 10.7 & 10.6 \\
\hline $\operatorname{rms}\left(A_{t}\right)^{d}$ & 4.2 & 3.4 & 3.0 & 6.2 & 4.9 & 4.3 \\
\hline \multicolumn{7}{|l|}{ Jerk time } \\
\hline $\operatorname{rms}\left(l_{j i j}\right)^{\mathrm{a}}$ & 4.8 & 4.6 & 4.5 & 6.0 & 5.6 & 5.5 \\
\hline $\operatorname{rms}\left(A_{i}\right)^{\mathrm{b}}$ & 2.6 & 2.1 & 1.9 & 3.1 & 2.5 & 2.3 \\
\hline $\operatorname{rms}\left(I_{t i}\right)^{c}$ & 5.2 & 4.9 & 4.8 & 6.0 & 5.6 & 5.5 \\
\hline $\operatorname{rms}\left(A_{t}\right)^{\mathrm{d}}$ & 2.8 & 2.2 & 2.0 & 3.1 & 2.6 & 2.3 \\
\hline
\end{tabular}

a Root-mean-square estimation error when the JEM model was used for estimating individual job exposure (see equation 4 and figure 2).

b Root-mean-square estimation error when the JEM model was used for estimating a group mean (see equation 5 and figure 3 ).

c Root-mean-square estimation error when the TEM model was used for estimating individual job exposure (see equation 4).

${ }^{d}$ Root-mean-square estimation error when the TEM model was used for estimating a group mean (see equation 5 ).
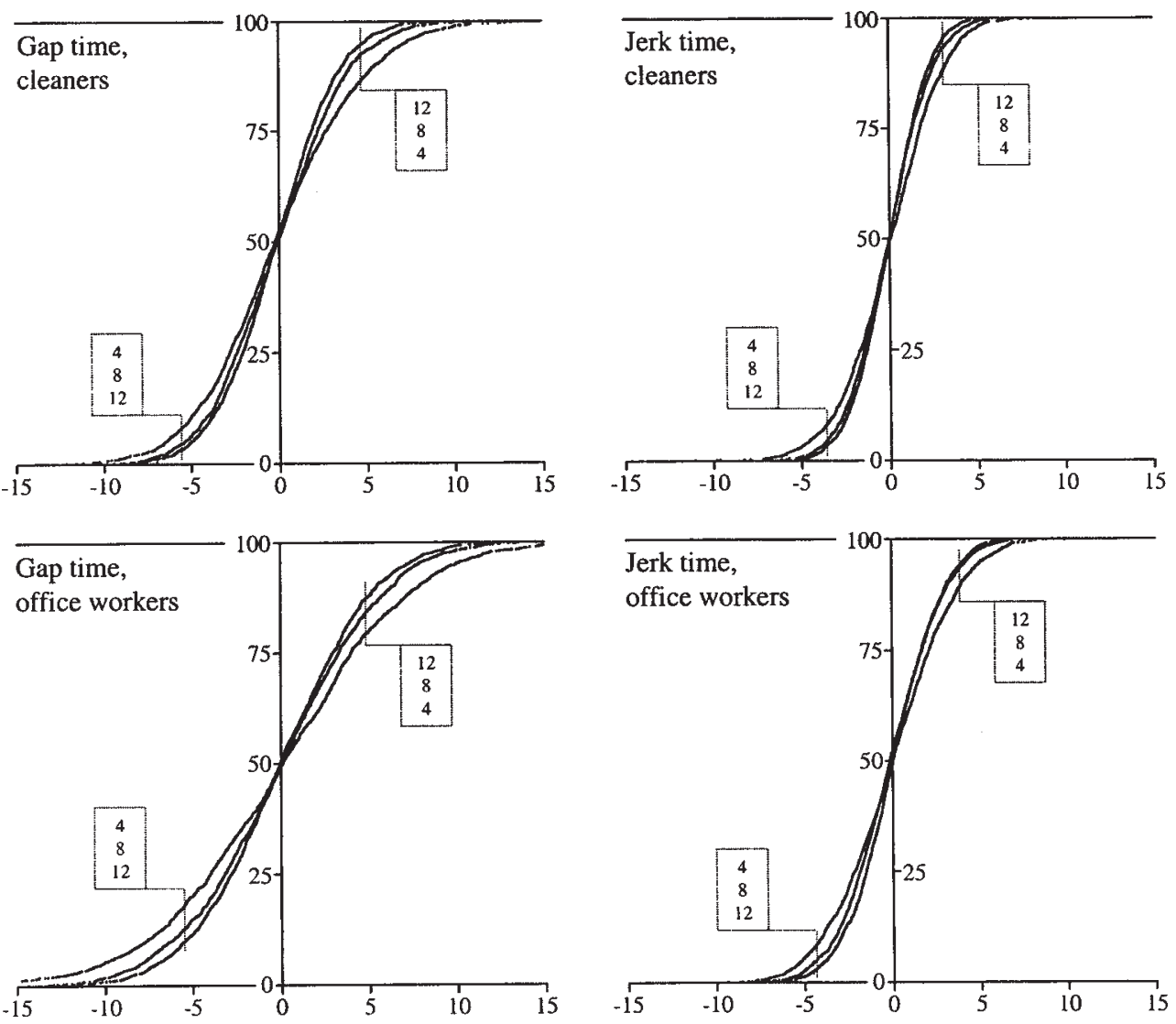

Correctness of the mean, JEM model; \%time

Figure 3. Empirical cumulative bootstrap distributions of the correctness of the job-exposure-matrix (JEM) estimates of group mean exposure (ie, $A_{j}$; equation 5). (text boxes and connected lines = locations of the distributions from top to bottom; $4,8,12=$ size of the database, $n_{b}$ ) 
in the size of the database (ie, $n_{b}$ ) led to a decreased probability of manifest disagreement between the JEM estimates and the true job-exposure means (figure 3). As for individual estimation correctness, the root-meansquare of $A_{j}$ closely agreed with analytical expectations, in this case based on equation 5 . The expected standard deviation of $A_{j}, s_{A}$, takes the form:

$$
s_{A}=\left(s_{S b}{ }^{2} / n_{b}+s_{S e}{ }^{2} / n_{e}\right)^{1 / 2},
$$

equation 9

where $n_{e}=$ the size of the target sample, notation otherwise as in equation 8 . As for individual estimates, $s_{S b}$ and $s_{S e}$ are both equal to $s_{S}$ (see table 1) in the present case.

\section{Task-exposure-matrix model}

The crude analysis of the ability of task-based estimates to predict job exposures in the parent dataset (table 1, regression results) suggested that they contained very little information beyond that already available on the basis of occupational group. This impression was substantiated by the crude analysis of estimation error (table 2) and by the more sophisticated performance analysis obtained through bootstrapping (figure 4 and table 3 for individual exposures, figure 5 and table 4 for group means).

Individual exposures. At the individual level, the estimation errors of the TEM model (table 2) indicated that it resulted in similar or slightly better estimates of job exposure than the JEM model in the office group. The gain analysis (table 3, figure 4) confirmed this finding by showing that the probability of getting an improved correctness by using the TEM model ranged from $49 \%$ to $53 \%$, depending on the exposure parameter and the size of the database, $n_{b}$. However, the differences between the TEM and JEM estimates were small. In contrast, among the cleaners, the TEM model performed, on the average, slightly worse than the JEM model (figure 4 , tables 2 and 3). In addition, a pronounced loss in correctness was more likely to occur than a gain of the same size (table 3). All of the examined relationships between JEM and TEM correctness were linear, the slopes ranging from 0.97 to 1.00 , intercepts between -0.40 and 0.24 , and an explained variance of between 0.81 and
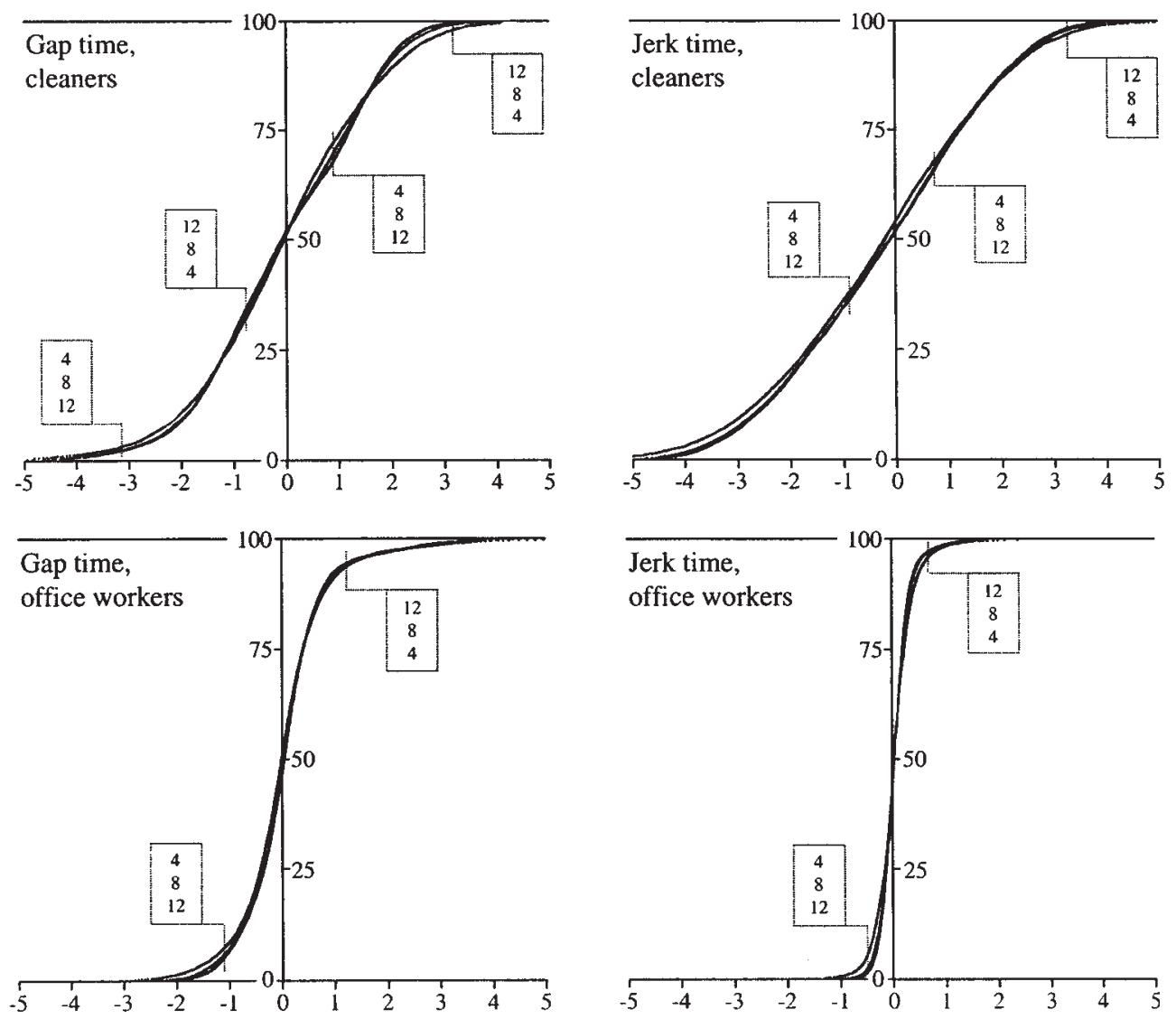

Gain in individual correctness; \%time

Figure 4. Empirical cumulative bootstrap distributions of the gain in individual correctness when the task-exposure-matrix (TEM) model was used instead of the job-exposure-matrix (JEM) model [ie, $G\left(I_{t i}\right)$, equation 6]. For clarity, the tails of some distributions have been omitted. (text boxes and connected lines $=$ the locations of the distributions from top to bottom; 4, 8, $12=$ size of the database, $n_{b}$ ) 
Table 3. Descriptive gain parameters when the task-exposurematrix (TEM) model was used for estimating individual job exposures instead of the job-exposure-matrix (JEM) model. Empirical probabilities (see figure 4) of obtaining an improved correctness and of the gain or loss in correctness being more than $2 \%$ time. The results are presented according to occupational group, exposure parameter, and size of the database $\left(n_{b}\right)$.

\begin{tabular}{|c|c|c|c|c|c|c|}
\hline & \multicolumn{3}{|c|}{ Cleaners } & \multicolumn{3}{|c|}{ Office workers } \\
\hline & $n_{b}=4$ & $n_{b}=8$ & $n_{b}=12$ & $n_{b}=4$ & $n_{b}=8$ & $n_{b}=12$ \\
\hline \multicolumn{7}{|l|}{ Gap time } \\
\hline $\begin{array}{l}\operatorname{Pr}\left[G\left(I_{t i}\right)>0\right]^{\mathrm{a}} \\
\operatorname{Pr}\left[G\left(I_{t_{t}}\right)>2\right]^{\mathrm{b}} \\
\operatorname{Pr}\left[G\left(I_{t i}\right)<-2\right]^{\mathrm{c}}\end{array}$ & $\begin{array}{l}48.8 \\
10.8 \\
10.9\end{array}$ & $\begin{array}{c}48.4 \\
8.8 \\
9.6\end{array}$ & $\begin{array}{l}48.1 \\
7.9 \\
9.2\end{array}$ & $\begin{array}{l}49.1 \\
2.7 \\
1.4\end{array}$ & $\begin{array}{l}52.2 \\
3.0 \\
0.4\end{array}$ & $\begin{array}{c}53.5 \\
2.9 \\
0.1\end{array}$ \\
\hline \multicolumn{7}{|l|}{ Jerk time } \\
\hline $\begin{array}{l}\operatorname{Pr}\left[G\left(I_{t i t}\right)>0\right]^{\mathrm{a}} \\
\operatorname{Pr}\left[G\left(I_{t i t}\right)>2\right]^{\mathrm{b}} \\
\operatorname{Pr}\left[G\left(I_{t_{i t}}\right)<-2\right]^{\mathrm{c}}\end{array}$ & $\begin{array}{l}46.0 \\
13.3 \\
20.4\end{array}$ & $\begin{array}{l}48.1 \\
13.0 \\
19.1\end{array}$ & $\begin{array}{l}48.0 \\
12.6 \\
18.7\end{array}$ & $\begin{array}{c}53.6 \\
0.1 \\
<0.1\end{array}$ & $\begin{array}{l}54.3 \\
<0.1 \\
<0.1\end{array}$ & $\begin{array}{l}54.8 \\
<0.1 \\
<0.1\end{array}$ \\
\hline
\end{tabular}

a Probability (in percent) that the TEM model improved the correctness at the individual level, as compared with the JEM model.

${ }^{b}$ Probability (in percent) that the gain in correctness at the individual level was larger than $2 \%$ time.

${ }^{c}$ Probability (in percent) that the gain in correctness at the individual level was less than $-2 \%$ time (ie, that the loss in correctness was larger than $2 \%$ time).
Table 4. Descriptive gain parameters when the task-exposure matrix (TEM) model was used for estimating group mean exposures instead of the job-exposure matrix (JEM) model. Empirical probabilities (see figure 5) of obtaining an improved correctness and of the gain or loss in correctness being more than $1 \%$ time. The results are presented according to occupational group, exposure parameter, and size of the database $\left(n_{b}\right)$.

\begin{tabular}{|c|c|c|c|c|c|c|}
\hline & \multicolumn{3}{|c|}{ Cleaners } & \multicolumn{3}{|c|}{ Office workers } \\
\hline & $n_{b}=4$ & $n_{b}=8$ & $n_{b}=12$ & $n_{b}=4$ & $n_{b}=8$ & $n_{b}=12$ \\
\hline \multicolumn{7}{|l|}{ Gap time } \\
\hline $\begin{array}{l}\operatorname{Pr}\left[G\left(A_{t}\right)>0\right]^{\mathrm{a}} \\
\operatorname{Pr}\left[G\left(A_{t}\right)>1\right]^{\mathrm{b}} \\
\operatorname{Pr}\left[G\left(A_{t}\right)<-1\right]^{\mathrm{c}}\end{array}$ & $\begin{array}{l}47.9 \\
10.7 \\
15.3\end{array}$ & $\begin{array}{c}47.4 \\
7.7 \\
10.8\end{array}$ & $\begin{array}{c}46.5 \\
7.5 \\
9.7\end{array}$ & $\begin{array}{c}46.6 \\
1.4 \\
4.2\end{array}$ & $\begin{array}{c}46.7 \\
0.8 \\
1.9\end{array}$ & $\begin{array}{c}45.8 \\
0.9 \\
1.4\end{array}$ \\
\hline \multicolumn{7}{|l|}{ Jerk time } \\
\hline $\begin{array}{l}\operatorname{Pr}\left[G\left(A_{t}\right)>0\right]^{\mathrm{a}} \\
\operatorname{Pr}\left[G\left(A_{t}\right)>1\right]^{\mathrm{b}} \\
\operatorname{Pr}\left[G\left(A_{t}\right)<-1\right]^{\mathrm{c}}\end{array}$ & $\begin{array}{l}44.0 \\
12.6 \\
20.4\end{array}$ & $\begin{array}{c}46.2 \\
9.4 \\
13.4\end{array}$ & $\begin{array}{c}44.5 \\
5.9 \\
12.4\end{array}$ & $\begin{array}{c}49.8 \\
0.2 \\
<0.1\end{array}$ & $\begin{array}{l}48.7 \\
<0.1 \\
<0.1\end{array}$ & $\begin{array}{l}48.3 \\
<0.1 \\
<0.1\end{array}$ \\
\hline
\end{tabular}

a Probability (in percent) that the TEM model improved the correctness of the group mean, as compared with the JEM model.

b Probability (in percent) that the gain in correctness of the mean was larger than $1 \%$ time.

c Probability (in percent) that the gain in correctness of the mean was less than $-1 \%$ time (ie, that the loss in correctness was larger than $1 \%$ time).
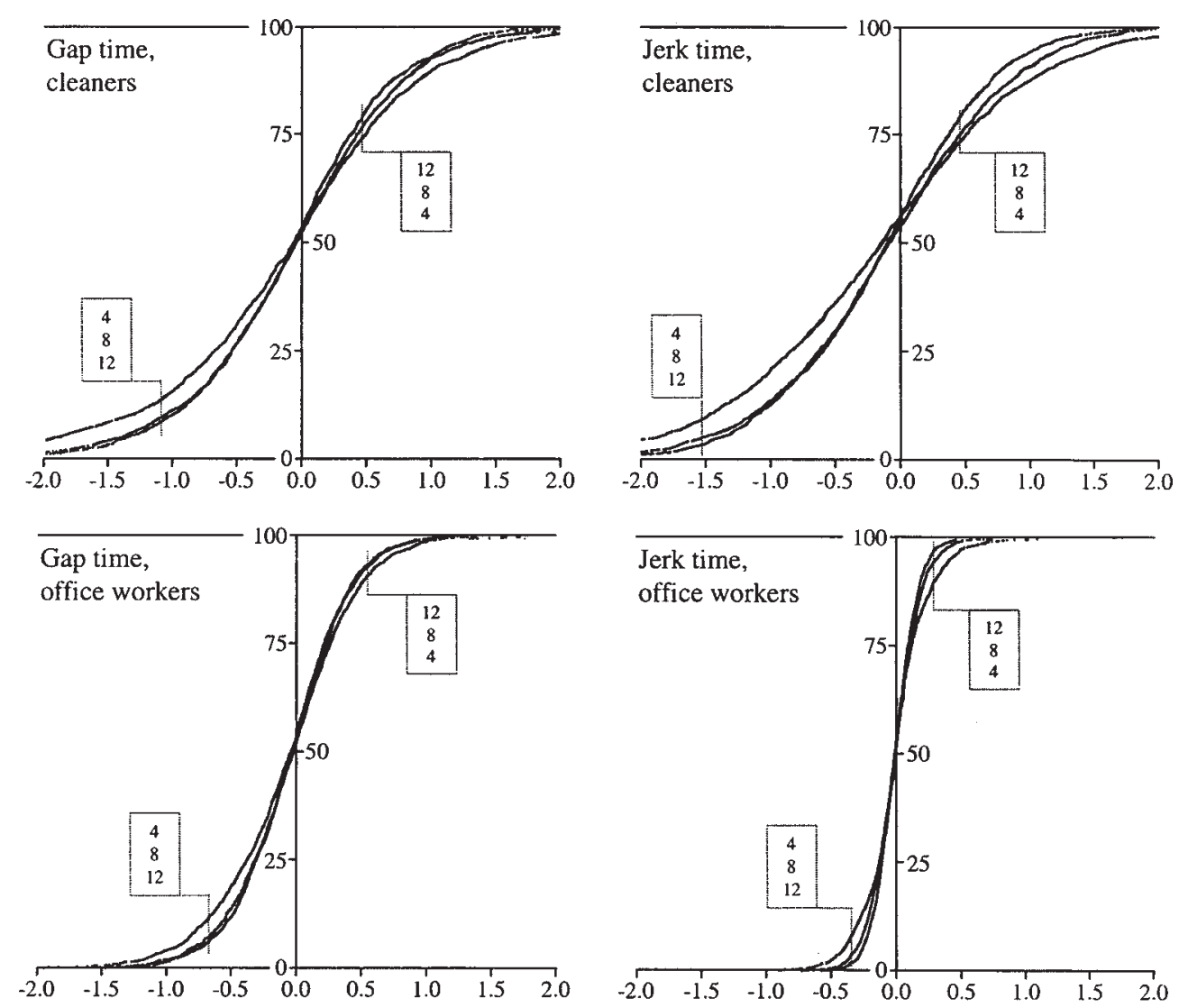

Gain in correctness of the mean; \%time

Figure 5. Empirical cumulative bootstrap distributions of the gain in correctness of the group mean when the task-exposure-matrix (TEM) model was used instead of the job-exposure-matrix (JEM) model [ie, $G\left(A_{t}\right)$; equation 7]. For clarity, the tails of some distributions have been omitted. (text boxes and connected lines $=$ the locations of the distributions from top to bottom; $4,8,12=$ size of the database, $n_{b}$ ) 
1.00. Thus no indications were found of the TEM model leading to increased or decreased correctness at any particular values of JEM correctness.

Group mean exposures. The TEM model led, in median, to slightly less correct mean exposures than the JEM model (tables 2 and 4, figure 5). Thus the probability that a TEM model would lead to a loss in correctness ranged from $51 \%$ to $56 \%$, depending on the exposure parameter and the size of the database, $n_{b}$ (table 4). Gain distributions were skewed so that cases of pronounced decrease in correctness were more frequent than cases of pronounced positive gain (table 4). As was found for individual correctness, the TEM exposure estimates were closer to the JEM estimates among the office workers than among the cleaners (figure 5, table 4).

\section{Discussion}

In our study, we estimated the mechanical job exposure of an individual by task exposures from a database weighted according to personal task proportions. We found only few and vague examples of the TEM model performing better than the JEM model, and several indications of performance loss, even when the job was composed of tasks with clearly contrasting exposures. Our finding, based on upper trapezius EMG, corroborates the results of a study of directly measured upperarm postures among machinists, car mechanics, and house painters (28). In that study, an equivalent of the TEM model was able to explain, at most, about $20 \%$ of the job-exposure variance between persons within each occupation, even though the tasks differed substantially in exposure. The claimed success of the task-based assessment of job energy expenditure in the study by Pernold et al (21) may well be explained preferentially by large exposure differences between the 21 occupations represented in the material. Thus a JEM approach might have been equally successful, yet without requiring extensive collection and processing of task information from all persons.

\section{Prospects of task-based estimation in the current settings}

Our study was based on recordings for an extended period of time and in a large parent set of persons as compared with most previous studies assessing upper trapezius EMG in an occupational setting [For reviews, see Mathiassen et al $(1,43)$.] Therefore, the data should be representative of the studied occupations, and the results therefore are not coincidental. As an inherent feature of the bootstrapping procedure, job- and task-exposure matrices were derived from persons within the same parent data set as the target persons on whom they were used. This approach favors successful exposure estimation, as compared with the use of job- and task-exposure matrices obtained from the literature.

The subjects differed substantially in job exposure in both occupational groups and for both EMG parameters $\left(\right.$ table $\left.1, s_{S}\right)(29)$. The variability in exposure was probably due to "true" differences between person means, as well as to differences between days within persons $(1,45)$. Since the EMG was measured for only 1 day per person, these sources of variability could not be separated. Because of the variability, the JEM model led to considerable inaccuracies (see figures 2 and 3 and tables 2 and 3) and showed a definite margin for improvement. Work was associated with significantly less gap time and more jerk time than breaks among both the cleaners and the office workers (table 1). The task-exposure contrast index $C_{T}$ also indicated that exposure discrimination was successful, at least in the cleaner's group (table 1). Thus, at this stage of information, corresponding to that available in the general literature on mechanical job and task exposures, we found no signs contradicting that TEM modeling could be a successful alternative to the JEM model. In addition, the overall exposure ranking of work and breaks was, in general, consistent at the level of the individual workers (figure 1), and task proportions did differ between persons (table 1, $s_{w t}$ ). The two latter pieces of information will not normally be available in a literaturebased TEM, but they confirmed what appeared to be a positive prognosis for task-based job-exposure modeling.

\section{Performance of task-based estimation in the current study}

Nevertheless, we found that the TEM model was, in general, no better than the JEM model in predicting job exposures. One potential source of decreased correctness of task-based estimates would be flawed task proportions. In the study, the activities of the persons were continuously observed and classified by an experienced ergonomist. While we do acknowledge that even trained observers may differ in their assessment, we believe that continuous observation is associated with smaller errors in task proportions than other, less meticulous methods based on self-reports. Still, erroneous task proportions may be a serious concern (46), leading to a diluted predictive ability of the task-based estimates or even systematic estimation bias (29).

The TEM model assumes that task exposures and task proportions are uncorrelated. We found clear examples, however, that a correlation was present, in particular for the cleaners, larger proportions of both work and breaks being associated with more gap time and less 
jerk time, indicating a more "rested" activity pattern (table $\left.1, r\left[w_{t} m_{t}\right]\right)$.

To investigate the importance of this correlation to the performance of the TEM model, we constructed a simple scenario including two tasks with exposure contrast $C_{T}=0.69$ (see equation 1), comparable to work and breaks among the cleaners (table 1). We then systematically varied the level of exposure-versus-proportion correlation by shuffling individual combinations of task proportion and task exposure. We found a general tendency for a larger correlation between the proportion and exposure of work to be associated with better performance of the TEM model relative to the JEM model. A larger correlation between task exposures (see $r\left[m_{w} m_{b}\right]$, table 1) was associated with better TEM model performance if the exposure-versus-proportion correlation for work was large, while the opposite was found for small values of $r\left[w_{t} m_{t}\right]$. In general, substantial differences in performance were observed even for scenarios with the same values of both $r\left[m_{w} m_{b}\right]$ and $r\left[w_{t} m_{t}\right]$. Thus we did not find any reason to believe that correlations between task exposures and task proportions, or between exposures in work and breaks, could explain why the TEM model was not successful in our settings.

Systematic relationships between the proportions and exposures of tasks could be integrated in extended task-based models for the estimation of job exposures. Similarly, models might be constructed on the basis of other empirical determinants of task exposures associated with the workplace (eg, work station dimensions, tool weights) or the worker (eg, age, anthropometry). We did not examine these options, and the necessary data will normally not be available if a task-exposure matrix is to be constructed on the basis of the literature.

Another alternative for refining task-based modeling is to replace the assumption of a one-to-one relationship between estimated and true job exposures expressed in the TEM model (see equation 2) by the empirical relationship, as determined by the regression of true job exposures on the TEM estimates (28). This approach is equivalent to a calibration of exposure surrogates as discussed elsewhere [eg, in nutrition epidemiology (47)]. As suggested by the outcomes of this regression in the parent data set (see table 1, regression), calibration would not be a viable alternative in our case. When examining the task-based regression model using bootstrapping, we even observed that substantial estimation errors were, in general, more likely than with the basic TEM model.

\section{Size of the database and target samples}

The size of the target sample, 10 persons, was chosen to diminish the likelihood of a major overlap of persons between that sample and the database sample, while still securing sufficient material for credible inferences. Since bootstrapping is based on re-sampling with replacement (41), the same person can be elected to both samples in a particular run, and the probability of this happening depends on the size of the samples. Partly for the same reason, the database size in the simulations, $n_{b}$, did not exceed 12 persons. Another reason for limiting $n_{b}$ was that a literature-based task-exposure matrix on occupational trapezius EMG would have to rely on studies that typically investigated fewer than 12 persons $(1,43)$.

Increasing the size of the database or the target sample would have led to closer agreement between the mean exposures in the two samples, and therefore a more compressed distribution of correctness when group means were estimated using the JEM model (see equation 9 and figure 3). However, for a database of size $n_{b}$, the JEM mean correctness cannot exceed that described by a normal distribution with a zero mean and standard deviation $\left(s_{S b}^{2} / n_{b}\right)^{1 / 2}$, where $s_{S b}$ stands for the overall standard deviation between subjects in true job exposure. This estimation error limit applies only if estimation is unbiased, as in our study, where the database and target samples were drawn from the same parent dataset. In the presence of bias, the estimation error increases. This increase can occur, for instance, if exposures in the target population are estimated using literature data from another setting, even if within the same occupation.

Correctness when individual exposures were estimated using the JEM model changed marginally with database size (see figure 2) and quickly approached limits set by the dispersion of individual job exposures in the database. This result agrees with equation 8 , which indicates that the exposure standard deviation between persons in the target sample, $s_{S e}$, largely determines the estimation error at "large" sizes of the database, $n_{b}$. In addition, equation 8 demonstrates that the error is not influenced by the size of the target sample, in contrast to the conditions when group means are estimated (see equation 9).

The performance of the TEM model relative to that of the JEM model was not affected much by the size of $n_{b}$ (see figures 4 and 5, tables 2 and 3 ) and cannot be expected to depend on the size of the target sample either. Thus we believe that the general results of the study are also valid for other sizes of the database and target samples.

In our investigation, task proportions were obtained for one workday of each person, and the performances of the JEM and TEM models were assessed using the true job exposure of that specific day. Due to the nature of the parent dataset, we were not able to investigate whether a task-based model would be effective in predicting the mean job exposure across multiple days with varying task proportions for a particular person. 
However, the correctness of both the JEM and the TEM models can be expected to increase if the models are used to estimate job exposures for prolonged periods, asymptotically approaching "lifetime". Correctness will improve because the inflating effect of the between-days within-subject exposure variability on the overall standard deviation $s_{S e}$ in the target population (see equations 8 and 9) vanishes as the estimated period increases. Probably, this effect will be distinct for EMG parameters, since their between-day variability seems to be considerable $(1,45)$. A time-extended TEM model requires task proportions covering prolonged periods of time, as obtained, for instance, by asking persons about the structure of a "typical" workday. This information may be uncertain and therefore lead to incorrect job-exposure estimates. The JEM model, on the other hand, does not suffer a corresponding risk of loss of input data quality, since "occupation" obviously does not depend on the number of days estimated. Therefore, we do not expect the performance of the TEM model to improve relative to that of the JEM model with a prolonged estimation period.

\section{Influence of the task classification scheme}

Our partition of jobs into work and breaks represented a coarse but unambiguous classification. The sizes of the within-task exposure variability between subjects (table $1, s_{S}$ ) were no larger than that reported in previous field studies of upper trapezius EMG during different occupational tasks $(39,48-51)$. In theory, a moredetailed subclassification of tasks within the job could entail improved exposure discrimination and thus offer a potential of more successful task-based modeling. The original task classification into five subcategories of work for the cleaners and four for the office workers was constructed to be exhaustive for the job and to be usable in self-assessment diaries (32). The subcategories were also expected to represent exposure contrasts, but they were not constructed specifically for that purpose, and subsequent analyses revealed that they were, in fact, inadequate with respect to both trapezius EMG $(29,30)$ and heart rate $(35)$.

In theory, a task classification scheme might have been developed that would better explain upper trapezius EMG variability during work than the one used by us. Since people are seemingly less able to report correct time proportions of "biomechanical" activities [eg, "hands above shoulders" $(52,53)]$ than job-contextual activities [eg, "sorting letters" $(54,55)]$, we believe that a successful task classification scheme for trapezius EMG could not be based on self-reports. What is left is the option of an observation-based and therefore resource-demanding classification related, for instance, to postures and work technique.
At the same time, introducing more task categories may exacerbate several sources of error that detract from the intended gains in exposure discrimination. First, more task categories increase the risk that task proportions will be inaccurate due to the reporter misinterpreting task descriptions or losing commitment (29). Second, increasingly detailed task specifications lower the likelihood that task exposures can be obtained from databases in the literature. We therefore believe that the inadequacy of the TEM model would not, for the present occupations, have been resolved by other, more-detailed task classification schemes based on observations or self-reports.

\section{Performance predictors for task-based estimation}

The impotence or even failure of task-based modeling of trapezius EMG (our study) and upper-arm postures (28) in various occupational groups emphasizes that the prognosis of modeling should be carefully considered before it is implemented in a particular epidemiologic study. Otherwise, there is a substantial risk that resources will be invested in obtaining and analyzing data at the task level with no return. Obvious prerequisites to effective task-based modeling are the existence of tasks that can be clearly identified and that differ in exposure, and a substantial differentiation between the workers with respect to task proportions. For reasons previously outlined, we emphasize that the task classification scheme should not be too detailed. While large contrasts in task proportions between workers are desirable, they may also indicate the possibility that workers can be correctly classified into "suboccupations". Then, within each suboccupation, individual job exposures can be estimated by the suboccupational mean, while detailed information on task proportions may not offer any additional gain in correctness. In general, task-based modeling is probably cost-effective only if task proportions or suboccupations are accessible with "cheap" methods, such as self-reports or production records (8).

As discussed previously, we only found weak indicators of the eventual performance of task-based modeling in the information normally available in a literaturebased task-exposure matrix (ie, task exposure means and within-task exposure variability). Theoretical considerations show that extreme values of the task contrast index $C_{T}$ correctly predict that TEM estimates of job exposure are perfect (at $C_{T}=1$ ) or completely uninformative (at $C_{T}=0$ ). In the, probably rare, cases in which such extremes are approached in an occupational setting, task-based modeling is therefore a viable option or a debacle, respectively. At less extreme values of $C_{T}$, the performance of the TEM model is not straightforwardly related to the size of $C_{T}$. An example of this situation is apparent when the observed values of $C_{T}$ in our study 
are compared with the predictive ability of the corresponding task-based estimates, as measured by the explained variance, $r^{2}$, in the regression of true job exposures on the estimates (table 1). Thus, in what appears to be the most common situation in occupational settings, the performance of a TEM model is strongly influenced by factors that the $C_{T}$ does not reflect; for instance, correlations between exposures in different tasks, correlations between task exposures and task proportions, and the dispersion of task proportions among the workers.

Thus far, empirical data are not available that can act as a guide for the general prognostic value of contrast indices, for instance, $C_{T}$, measures of proportion diversity, for example, $s_{w t}$ (table 1), or parameters combining them, such as the product of $s_{w t}$ and $C_{T}$, or the between-subject variability of task-based estimates in a pilot dataset. As noted earlier, correlations between proportions and exposures of individual tasks or between exposures in different tasks do not appear to be straightforward indicators of the relative performance of the TEM model either.

Thus a pilot study providing information on task proportions, task exposures, and true job exposures will often be needed in a particular occupational setting to reveal whether task-based modeling is, indeed, a viable option. By means of work sampling (56-58) or continuous whole-day exposure recordings (as in our dataset), accompanied by an extensive collection of information as to activities in the job, data can be obtained that allow an analysis of the performance of different task classification schemes. A crude regression analysis of true job exposures on TEM estimates (table 1, regression parameters) can then reveal whether these estimates appear informative, and, to a certain extent, whether they seem correct for group means and individual job exposures. A detailed understanding of the performance of different modeling alternatives can be obtained by simulations similar to those used in our study.

We speculate that task-based modeling can be effective for some exposure parameters in some occupational settings in which contrasts between tasks are larger than in the present case, task proportions are more diverse, and correlations between task proportions and task exposures are favorable. One example tentatively supported by some previous studies $(24,26)$, although distrusted by others (59), is that low-back loads may be acceptably estimated for occupations in which some persons are engaged in heavy manual handling while others are not.

In addition to the epidemiologic application investigated by us, a task-based approach is often used in ergonomic practice for identifying the hazardous job elements, which are then targets for intervention. The use of a task-based strategy for this purpose implicitly assumes that, for instance, a decrease in the proportion of a high-exposure task will result in a reduced job exposure. An equivalent approach has been used for predicting the result of major redistributions of tasks between subjects, such as in job rotation scenarios (27). In these cases, task-based modeling is used in an attempt to estimate the direction of a job exposure change as opposed to the application investigated in our study, which aimed at estimating job-exposure levels of workers and groups. Our data illustrate that, while the latter approach may be highly unsuccessful, the former can, even in the same setting, be effective. Thus figure 1 suggests that a larger proportion of work and, consequently, fewer breaks would, for most cleaners, result in less gap time and more jerk time at the job level.

\section{Concluding remarks}

Task-based estimates of job exposure were, in general, less correct than estimates based on a job-exposure matrix. The performance of our task-based model could not be predicted on the basis of standard exposure data normally available in the literature. We, therefore, caution against the common belief in occupational epidemiology that task-based modeling is a cost-effective approach to the estimation of mechanical job exposures, and we recommend that thorough pilot studies be conducted in any specific case. We do not exclude, however, that task information can be valuable for predicting job exposure in other occupational groups and for other parameters than those investigated in our study. In addition, task-based modeling may be effective in common applications other than job-exposure estimation, for instance, in predicting the effects of reducing high-exposure tasks in a job.

\section{Acknowledgments}

Parts of our study were supported by the Swedish Council for Work Life Research, the Swedish Councils for Planning and Coordination of Research, the Medical Faculty of Lund University, and the County Councils of Southern Sweden.

Donald Halstead, Harvard School of Public Health, is gratefully acknowledged for his valuable editorial suggestions.

\section{References}

1. Mathiassen SE, Burdorf A, van der Beek AJ. Statistical power and measurement allocation in ergonomic intervention studies assessing upper trapezius EMG amplitude: a case study of assembly work. J Electromyogr Kinesiol 2002;12:27-39. 
2. Mathiassen SE, Möller T, Forsman M. Variability in mechanical exposure within and between individuals performing a highly constrained industrial work task. Ergonomics 2003; 46:800-24.

3. Tielemans E, Kupper LL, Kromhout H, Heederik D, Houba R. Individual-based and group-based occupational exposure assessment: some equations to evaluate different strategies. Ann Occup Hyg 1998;42:115-9.

4. Reeves GK, Cox DR, Darby SC, Whitley E. Some aspects of measurement error in explanatory variables for continuous and binary regression models. Stat Med 1998;17:2157-77.

5. Loomis D, Kromhout H. Exposure variability: concepts and applications in occupational epidemiology. Am J Ind Med 2004;45:113-22.

6. Winkel J, Mathiassen SE. Assessment of physical work load in epidemiologic studies: concepts, issues and operational considerations. Ergonomics 1994;37:979-88.

7. van der Beek AJ, Frings-Dresen MHW. Assessment of mechanical exposure in ergonomic epidemiology. Occup Environ Med 1998;55:291-9.

8. Burdorf A, van der Beek A. Exposure assessment strategies for work-related risk factors for musculoskeletal disorders. Scand J Work Environ Health 1999;25 suppl 4:25-30.

9. Hagberg M, Punnett L, Bergqvist U, Burdorf A, Härenstam A, Kristensen TS, et al. Broadening the view of exposure assessment [consensus report]. Scand J Work Environ Health 2001;27:354-7.

10. Burdorf A, Rossignol M, Fathallah FA, Snook SH, Herrick RF. Challenges in assessing risk factors in epidemiologic studies on back disorders. Am J Ind Med 1997;32:142-52.

11. Christmansson M, Medbo L, Hansson G-Å, Ohlsson K, Unge Byström J, Möller T, et al. A case study of a principally new way of materials kitting - an evaluation of time consumption and physical workload. Int J Ind Ergon 2002;30:49-65.

12. Benke G, Sim M, Fritschi L, Aldred G. Beyond the job exposure matrix (JEM): the task exposure matrix (TEM). Ann Occup Hyg 2000;44:475-482.

13. Goldberg M, Kromhout H, Guénel P, Fletcher AC, Gérin M, Glass DC, et al. Job exposure matrices in industry. Int $\mathrm{J}$ Epidemiol 1993;22 suppl 2:S10-S15.

14. Kauppinen T, Toikkanen J, Pukkala E. From cross-tabulations to multi-purpose exposure information systems: a new job exposure matrix. Am J Ind Med 1998;33:409-17.

15. Hansen DJ, Whitehead LW. The influence of task and location on solvent exposures in a printing plant. Am Ind Hyg Assoc J 1988;49:259-65.

16. Olsen E. Analysis of exposure using a logbook method. Appl Occup Environ Hyg 1994;9:712-22.

17. Corn M, Esmen NA. Workplace exposure zones for classification of employee exposures to physical and chemical agents. Am Ind Hyg Assoc J 1979;40:47-57.

18. Harrison RM, Thornton CA, Lawrence RG, Mark D, Kinnersley RP, Ayres JG. Personal exposure monitoring of particulate matter, nitrogen dioxide, and carbon monoxide, including susceptible groups. Occup Environ Med 2002;59:671-9.

19. Preller L, Kromhout H, Heederik D, Tielen MJM. Modeling long-term average exposure in occupational exposure-response analysis. Scand J Work Environ Health 1995;21:504-12.

20. Bernard TE, Joseph BS. Estimation of metabolic rate using qualitative job descriptors. Am Ind Hyg Assoc J 1994; 55:1021-9.

21. Pernold G, Wigaeus Hjelm E, Wiktorin C, Mortimer M, Karlsson E, Kilbom $\AA$, et al. Validity of occupational energy expenditure assessed by interview. Am Ind Hyg Assoc J

\section{2;63:29-33.}

22. Bongers PM, Hulshof CTJ, Dijkstra L, Boshuizen HC, Groenhout HJM, Valken E. Back pain and exposure to whole-body vibration in helicopter pilots. Ergonomics 1990;33:1007-26.

23. Liu WCV, Sanchez-Monroy D. Prevalence of back discomfort and estimates of back load in two manufacturing facilities. Ann Occup Hyg 1998;42:549-56.

24. Burdorf A, van Riel M, Brand T. Physical load as risk factor for musculoskeletal complaints among tank terminal workers. Am Ind Hyg Assoc J 1997;58:489-97.

25. Frost P, Bonde JP, Mikkelsen S, Andersen JH, Fallentin N, Kaergaard A, et al. Risk of shoulder tendinitis in relation to shoulder loads in monotonous repetitive work. Am J Ind Med 2002;41:11-8

26. Norman R, Wells R, Neumann P, Frank J, Shannon H, Kerr $\mathrm{M}$, et al. A comparison of peak vs cumulative physical work exposure risk factors for the reporting of low back pain in the automotive industry. Clin Biomech 1998;13:561-73.

27. Frazer MB, Norman RW, Wells RW, Neumann WP. The effects of job rotation on the risk of reporting low back pain. Ergonomics 2003;46:904-19.

28. Svendsen SW, Mathiassen SE, Bonde JP. Task-based exposure assessment in ergonomic epidemiology — a study of upper arm elevation in the jobs of machinists, car mechanics, and house painters. Occup Environ Med 2005;62:18-26.

29. Mathiassen SE, Burdorf A, van der Beek AJ, Hansson G- $\AA$. Efficient one-day sampling of mechanical job exposure dataa study based on upper trapezius activity in cleaners and office workers. Am Ind Hyg Assoc J 2003;64:196-211.

30. Nordander C, Hansson G-Å, Rylander L, Asterland P, Unge Byström J, Ohlsson K, et al. Muscular rest and gap frequency as EMG measures of physical exposure: the impact of work tasks and individual related factors. Ergonomics 2000; 43:1904-19.

31. Hansson G-Å, Nordander C, Asterland P, Ohlsson K, Strömberg U, Skerfving S, et al. Sensitivity of trapezius electromyography to differences between work tasks-influence of gap definition and normalisation methods. J Electromyogr Kinesiol 2000;10:103-15.

32. Hansson G- $\AA$, Balogh I, Unge Byström J, Ohlsson K, Nordander C, Asterland P, et al. Questionnaire versus direct technical measurements in assessing postures and movements of head, upper back, arms and hands. Scand J Work Environ Health 2001;27(1):30-40.

33. Hansson G- $\AA$, Asterland P, Kellerman M. Modular data logger system for physical work load measurement. Ergonomics 2003;46:407-415.

34. Hansson G- $\AA$, Asterland P, Skerfving S. Acquisition and analysis of whole-day electromyographic field recordings. In: Hermens HJ, Hägg G, Freriks B, editors. European applications of surface electromyography. Stockholm: SENIAM / Roessingh Research and Development; 1997. p 19-27.

35. Balogh I, Orbak P, Ohlsson K, Nordander C, Unge J, Winkel $\mathrm{J}$, et al. Self-assessed and directly measured occupational physical activities-influence of musculoskeletal complaints, age and gender. Appl Ergon 2004;35:49-56.

36. Mathiassen SE, Winkel J. Quantifying variation in physical load using exposure-vs-time data. Ergonomics 1991;34:1455-68.

37. Jensen C, Nilsen K, Hansen K, Westgaard R. Trapezius muscle load as a risk indicator for occupational shoulder-neck complaints. Int Arch Occup Environ Health 1993;64:415-23.

38. Veiersted KB, Westgaard RH, Andersen P. Electromyographic evaluation of muscular work pattern as a predictor of trapezius myalgia. Scand J Work Environ Health 1993;19:284- 
290.

39. Hägg GM, Åström A. Load pattern and pressure pain threshold in the upper trapezius and psychosocial factors in medical secretaries with and without shoulder/neck disorders. Int Arch Occup Environ Health 1997;69:423-32.

40. Kirk RE. Experimental design: procedures for the behavioral sciences. Pacific Grove (CA): Brooks/Cole Publishing Company; 1995.

41. Diaconis P, Efron B. Computer-intensive methods in statistics. Sci Am 1983;May issue:96-108.

42. Hoozemans MJM, Burdorf A, van der Beek AJ, Frings-Dresen MHW, Mathiassen SE. Group-based measurement strategies in exposure assessment explored by bootstrapping. Scand J Work Environ Health 2001;27:125-32.

43. Mathiassen SE, Winkel J, Hägg G. Normalization of surface EMG amplitude from the upper trapezius muscle in ergonomic studies—a review. J Electromyogr Kinesiol 1995;5:195-226.

44. Samuels SJ, Lemasters GK, Carson A. Statistical methods for describing occupational exposure measurements. Am Ind Hyg Assoc J 1985;46:427-33.

45. Nordander C, Balogh I, Mathiassen SE, Ohlsson K, Unge J, Skerfving S, et al. Precision of measurements of physical workload during standardised manual handling, part I: surface electromyography of $\mathrm{m}$. trapezius, $\mathrm{m}$. infraspinatus and the forearm extensors. J Electromyogr Kinesiol 2004;14:443-54.

46. Homan MM, Armstrong TJ. Evaluation of three methodologies for assessing work activity during computer use. Am Ind Hyg Assoc J 2003;64:48-55.

47. Rosner B, Willett WC, Spiegelman D. Correction of logistic regression relative risk estimates and confidence intervals for systematic within-person measurement error. Stat Med 1989;8:1051-69.

48. Veiersted KB, Westgaard RH. Development of trapezius myalgia among female workers performing light manual work. Scand J Work Environ Health 1993;19:277-83.

49. Fernström EAC, Åborg CM. Alterations in shoulder muscle activity due to changes in data entry organisation. Int $\mathrm{J}$ Ind Ergon 1999;23:231-40.

50. Jensen C, Finsen L, Hansen K, Christensen H. Upper trapezi- us muscle activity patterns during repetitive manual material handling and work with a computer mouse. J Electromyogr Kinesiol 1999;9:317-25.

51. Westgaard RH, Vasseljen O, Holte KA. Trapezius muscle activity as a risk factor for shoulder and neck pain in female service workers with low biomechanical exposure. Ergonomics 2001;44:339-53.

52. Mortimer M, Wigaeus Hjelm E, Wiktorin C, Pernold G, Kilbom $\AA$, Vingård E, et al. Validity of self-reported duration of work postures obtained by interview. Appl Ergon 1999; 30:477-86.

53. Spielholz P, Silverstein B, Morgan M, Checkoway H, Kaufman J. Comparison of self-report, video observation and direct measurement methods for upper extremity musculoskeletal disorder physical risk factors. Ergonomics 2001;44:588613.

54. Petersson NF, Mathiassen SE, Björing G, Winkel J. The accuracy of self-rating of exposure to repetitive work. Int $\mathrm{J}$ Ind Ergon 2000;25:239-46.

55. van der Beek AJ, Braam I, Douwes M, Bongers P, FringsDresen M, Verbeek J, et al. Validity of a diary estimating exposure to tasks, activities, and postures of the trunk. Int Arch Occup Environ Health 1994;66:173-8.

56. Buchholz B, Paquet V, Punnett L, Lee D, Moir S. PATH: a work sampling-based approach to ergonomic job analysis for construction and other non-repetitive work. Appl Ergon 1996;27:177-87.

57. Frings-Dresen MHW, Kuijer PPFM. The TRAC-system: an observation method for analyzing work demands at the workplace. Safety Sci 1995;21:163-5.

58. Neumann WP, Wells RP, Norman RW, Frank J, Shannon H, Kerr MS, et al. A posture and load sampling approach to determining low-back pain risk in occupational settings. Int J Ind Ergon 2001;27:65-77.

59. Hye-Knudsen CT, Schibye B, Hjortskov N, Fallentin N. Trunk motion characteristics during different patient handling tasks. Int J Ind Ergon 2004;33:327-37.

Received for publication: 27 November 2003 